\title{
INFECCIÓN URINARIA NO COMPLICADA EN MUJERES: ETIOLOGÍA Y RESISTENCIA A ANTIMICROBIANOS
}

\author{
UNCOMPLICATED URINARY TRACT INFECTION IN WOMEN: \\ ETIOLOGY AND ANTIMICROBIAL RESISTANCE
}

Diego Federico Baenas ${ }^{1}$, Hugo J Palmieri², Juan Manuel Alomar ${ }^{3}$, Joaquín Horacio Álvarez Garzón ${ }^{4}$, Leandro Berenguer ${ }^{5}$, Mario Vilaró ${ }^{6}$, Ricardo Arturo Albertini ${ }^{7}$

\section{Resumen:}

Nuestros objetivos fueron determinar la etiología y analizar los perfiles de resistencia antimicrobiana de los microorganismos causantes de infecciones urinarias no complicadas en nuestro medio. Se realizó un estudio analítico de corte transversal. Se analizó la resistencia antimicrobiana in vitro de los urocultivos.

Se incluyeron 580 urocultivos de mujeres mayores de 15 años.

Un $82.6 \%$ de urocultivos correspondieron a cistitis y el $17.4 \%$ a pielonefritis.

Se obtuvieron 353 urocultivos de mujeres < 50 años (60.9\%) y 227 a $\geq 50$ años $(39.1 \%)$.

Los patógenos más frecuentes fueron: Escherichia coli (85.5\%) y Klebsiella pneumoniae (4.7\%). Se encontró una resistencia de E coli a trimetoprima-sulfametoxazol del $28.6 \%$, a ciprofloxacina de $7.9 \%$ y a nitrofurantoína de $0.4 \%$.

Se evidenció diferencia significativa $(p=0.005)$ en la resistencia de $E$ coli a ciprofloxacina en las mujeres $\geq 50$ años de edad.

Nuestros datos muestran que existe una baja resistencia in vitro a nitrofurantoína.

Palabras clave: cistitis, pielonefritis, resistencia a antibióticos.

\section{Abstract:}

Our objectives were to determine the etiology and analyze the antibiotic resistance profiles of microorganisms causing uncomplicated urinary tract infections in our setting.

An analytical cross-sectional study was conducted. In vitro antimicrobial resistance of urine cultures was analyzed.

580 urine cultures of women over age fifteen were included.

$82.6 \%$ of urine cultures corresponded to cystitis and the remaining $17.4 \%$ corresponded to pyelonephritis. 353 urine cultures of women $<50$ years old $(60.9 \%)$ and 227 of women $\geq 50$ years old $(39.1 \%)$ were obtained.

The most common pathogens were Escherichia coli (85.5\%) and Klebsiella pneumoniae (4.7 \%). For Escherichia coli, there was a resistance of $28.6 \%$ to trimethoprim-sulfamethoxazole,7.9\% to ciprofloxacin and $0.4 \%$ to nitrofurantoin.

Significant difference $(p=0.005)$ was seen in the resistance to ciprofloxacin in women $\geq 50$ years old.

Our data show there is a low in vitro resistance to nitrofurantoin.

Key words: cystitis, pyelonephritis, antibiotic resistance.

\footnotetext{
Diego Federico Baenas: Hospital Privado Centro Médico de Córdoba.

Hugo J Palmieri: Hospital Privado Centro Médico de Córdoba.

Juan Manuel Alomar: Hospital Privado Centro Médico de Córdoba.

Joaquín Horacio Álvarez Garzón: Hospital Privado Centro Médico de Córdoba.

Leandro Berenguer: Hospital Privado Centro Médico de Córdoba.

Mario Vilaró: Hospital Privado Centro Médico de Córdoba.

Ricardo Arturo Albertini: Hospital Privado Centro Médico de Córdoba.
} 


\section{Introducción}

La infección del tracto urinario (IU) no complicada es aquella que ocurre en mujeres, previamente sanas, sin historia de anormalidad estructural o funcional de las vías urinarias. Se excluyen de esta clasificación a los hombres y niños, y a aquellas mujeres que presentan condiciones metabólicas, anatómicas o funcionales que puedan aumentar el riesgo de fracaso del tratamiento (por ejemplo, obstrucción del tracto urinario, litiasis, embarazo, diabetes, vejiga neurogénica, insuficiencia renal, inmunosupresión) $)^{(1)}$.

Entre 40 y $60 \%$ de las mujeres adultas refieren haber tenido al menos una IU a lo largo de su vida(2)

La mayoría de las IU son tratadas en forma empírica debido a que los patógenos involucrados son altamente predecibles. Sin embargo en los últimos años se ha reportado un cambio progresivo en la resistencia a los antibióticos más utilizados, con diferencias notables entre países, ciudades, e incluso entre centros estudiados ${ }^{(3-7)}$.

Existen pocos datos disponibles en nuestra ciudad y país. En este sentido, se destaca el trabajo de Rossi y colaboradores, en el que participaron 23 laboratorios de instituciones hospitalarias públicas y privadas de Argentina, en el período comprendido entre los años 1995 y 1996. Allí se exponen los porcentajes de resistencia a los antimicrobianos de microorganismos causantes de IU en pacientes hospitalizados y ambulatorios ${ }^{(8)}$.

Sin embargo, en ese estudio, no se analizaron por separado los urocultivos correspondientes a pacientes con IU complicadas y no complicadas.

Con respecto a este postulado, Lopardo G. y colaboradores, en el estudio Vigi-A/SADI realizado por la Sociedad Argentina de Infectología, observaron que existían diferencias al comparar las tasas de resistencia a Trimetoprima Sulfametoxazol (SMX) en la ciudad de Buenos Aires, en relación a las informadas por los sistemas de vigilancia (Estudio SIR - Sistema Informatizado de Resistencia), ya que estos últimos tampoco discriminaban infecciones complicadas de no complicadas ${ }^{(9)}$.

El presente trabajo describe la etiología y los perfiles de resistencia a antimicrobianos de las IU no complicadas en la población ambulatoria de mujeres adultas atendidas en el Hospital Privado Centro Médico de Córdoba. Además, analizamos las diferencias existentes entre mujeres mayores y menores de 50 años y entre las IU altas y bajas. Por último, comparamos nuestros resultados con los del estudio Vigi-A/SADI.

\section{Material y Métodos}

Se realizó un estudio analítico de corte transversal. Nuestro hospital es una institución privada, de tercer nivel de atención, que cuenta con un edificio central y 6 centros de atención ambulatoria ubicados en distintos puntos de Córdoba Capital. Cuenta con un sistema de historia clínica electrónica mediante el cual se realizan los pedidos de urocultivo. Con la finalidad de contar con muestras para este trabajo, se organizaron reuniones informativas con los médicos de los diferentes servicios del Hospital que solicitan urocultivos con mayor frecuencia y se propuso que se solicitara este método diagnóstico a aquellas pacientes que presentaran síntomas urinarios bajos o altos. Se desarrolló un cuestionario que aparecía en una ventana desplegable cada vez que un médico solicitaba un urocultivo en cualquier área del hospital. El cuestionario constaba de 11 preguntas diseñadas para discriminar diferentes subgrupos de pacientes según factores de riesgo para IU de diferentes etiologías y/o con distintos perfiles de resistencia antibiótica.

El Departamento de Cómputos desarrolló un sistema informático que integraba en una base de datos todo urocultivo informado como positivo, su etiología, el perfil de resistencia, fecha, los datos personales de los pacientes obtenidos de la historia clínica electrónica, y la información recabada al momento de la solicitud. Semanalmente se realizó un control y seguimiento a los fines de completar cualquier dato faltante mediante llamado telefónico.

Se incluyeron los urocultivos positivos de pacientes ambulatorios de sexo femenino mayores a 15 años realizados en el Laboratorio de Microbiología del Hospital Privado desde el 1 de septiembre de 2009 hasta el 30 de setiembre de 2011.

Se excluyeron los urocultivos pertenecientes a pacientes con: diabetes mellitus, embarazo, internación en el último mes, antibioticoterapia en el último mes, más de tres infecciones urinarias en el último año, alguna alteración por causa urológica, litiasis urinaria, institucionalizados, sondaje uretral o procedimiento urológico en los últimos 30 días, tratamiento dialítico, inmunosupresión y/o quimioterapia. También se excluyeron los pacientes de sexo masculino y los urocultivos solicitados en pacientes asintomáticos (pacientes que iban a ser sometidos a procedimientos invasivos de la vía urinaria).

Se dividieron las pacientes según edad en $<50$ años $y \geq 50$ años. 
En concordancia con la literatura (1), incluimos en el grupo "cistitis" a aquellas pacientes que se presentaron a la consulta con síntomas de disuria, con o sin polaquiuria, urgencia miccional o dolor suprapúbico, y, en el grupo "pielonefritis", si presentaban fiebre (temperatura> $38{ }^{\circ} \mathrm{C}$ ), escalofríos, dolor lumbar, dolor a la puño percusión del ángulo costo-vertebral y náuseas o vómitos, con o sin síntomas urinarios bajos.

Las muestras de orina fueron tomadas mediante la técnica de chorro medio.

El Laboratorio de Microbiología utiliza como método de rutina la técnica automatizada de Vitek® para determinar la sensibilidad antimicrobiana in vitro a través de la concentración inhibitoria mínima, y también para la identificación de especie. El laboratorio se encuentra integrado al programa de control de calidad externo de pruebas de sensibilidad a antibióticos del Instituto de Salud "Dr. Carlos Malbrán".

Se analizó la resistencia microbiana a los antibióticos más utilizados como terapia empírica: ampiclina, ampicilina-sulbactam, cefalexina, trimetoprima-sulfometoxazol, nitrofurantoína y ciprofloxacina.

El diagnóstico de IU se estableció ante el aislamiento de un solo uropatógeno con más de $100000 \mathrm{UFC} / \mathrm{ml}$ conforme a los criterios de la Infectious Diseases Society of America(10). Se descartaron las muestras contaminadas (menores a $100000 \mathrm{UFC} / \mathrm{ml}$ o con crecimiento mixto).

Se calculó media y desvío estándar para la variable edad. Se realizó análisis de las variables discretas con $\mathrm{X} 2$ tomando como significativa una $\mathrm{p} \leq 0.05$ con un intervalo de confianza del $95 \%$. Los datos fueron procesados mediante el programa STATA versión 11.

El trabajo fue aprobado por el Comité de Bioética de nuestro hospital.

\section{Resultados}

De un total de 1826 urocultivos positivos, se obtuvieron 580 que cumplían los criterios de inclusión (un urocultivo por paciente). La edad promedio fue 45 años (17-88 años, DS \pm 19.3 años).353 urocultivos correspondieron $a<50$ años $(60.9 \%)$ y 227 a $\geq 50$ años (39.1\%).

Se encontraron 13 tipos de uropatógenos.

En orden de frecuencia, estos fueron: Escherichia coli, $\mathrm{n}=496$ (85.5\%); Klebsiella pneumoniae, $\mathrm{n}=27$ $(4.7 \%) ; \quad$ Staphylococcus saprophyticus, $n=18$ (3.1\%); Proteus mirabilis, $n=15 \quad(2.6 \%)$; Enterococcus faecalis, $n=6$ (1\%); Staphylococcus aureus, $n=6 \quad(1 \%)$, Staphylococcus coagulasa negativo, $\quad n=3 \quad(0.5 \%)) ; \quad$ Staphylococcus haemolyticus, $\mathrm{n}=2(0.3 \%)$; Enterobacter $\mathrm{spp}, \mathrm{n}=2$ $(0.3 \%)$; Citrobacter freundii, $\mathrm{n}=2(0.3 \%)$; Klebsiella oxytoca, $n=1(0.2 \%)$ Enterobacter aerogenes, $n=1$ $(0.2 \%)$ y Proteus vulgaris $n=1(0.2)$.

Las 4 bacterias más frecuentes (E. coli, K. pneumoniae, S. saprophyticus y P. mirabilis) corresponden al $95.9 \%$ de la muestra.

Los perfiles de resistencia antibiótica hallados se detallan en la Tabla 1.

\begin{tabular}{|c|c|c|c|c|c|c|c|c|}
\hline \multirow{2}{*}{$\begin{array}{l}\text { Microorganis } \\
\text { mo }\end{array}$} & \multirow[b]{2}{*}{$\mathrm{N}$} & \multirow[b]{2}{*}{$\%$} & \multicolumn{6}{|c|}{ Resistencia antimicrobiana* } \\
\hline & & & $\mathrm{AM}$ & AMS & CFX & SMX & $\mathrm{NF}$ & CPX \\
\hline E. coli & 496 & 85.5 & 47.8 & 19.6 & 9.5 & 28.6 & 0.4 & 7.9 \\
\hline K.pneumonia & 27 & 4.7 & 100 & 25.9 & 11.1 & 14.8 & 18.5 & 0 \\
\hline $\begin{array}{l}\text { S. } \\
\text { saprophyticus }\end{array}$ & 18 & 3.1 & - & - & 0 & 0 & 0 & 0 \\
\hline $\begin{array}{l}\text { Proteus } \\
\text { mirabilis }\end{array}$ & 15 & 2.6 & 6.7 & 0 & 0 & 20 & 100 & 0 \\
\hline Otros & 24 & 4.1 & 37.5 & 33.3 & 25 & 0 & 12.5 & 0 \\
\hline Total & 580 & 100 & & & & & & \\
\hline $\begin{array}{l}\mathrm{AM}=\text { Ampic } \\
\text { ulfometoxazol }\end{array}$ & Tit & $\begin{aligned} \text { An } \\
\text { ntoin }\end{aligned}$ & 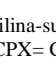 & flox, & $\mathrm{K}=$ & xina & $=\mathrm{T}_{1}$ & toprima \\
\hline
\end{tabular}

Del total de los urocultivos positivos, el $82.6 \%$ $(n=479)$ correspondió a IU bajas y el $17.4 \%$ $(n=101)$ a IU altas.

De las mujeres $<50$ años, $78.8 \%$ presentaron sintomatología de IU baja, y $21.2 \%$ de IU alta. En el grupo de $\geq 50$ años, en cambio, el $85 \%$ tenían síntomas de IU baja, y el $15 \%$ restante de IU alta.

Al comparar la prevalencia de los uropatógenos entre los grupos etarios, sólo se observaron diferencias significativas en el grupo "otros", constituido por microorganismos aislados y de muy baja prevalencia en este tipo de infecciones. (Tabla 2).

Tabla 2: Prevalencia de microorganismos más frecuentes según edad.

\begin{tabular}{lccc}
\hline \multirow{2}{*}{ Microorganismo } & \multicolumn{3}{c}{ Prevalencia(n, \%) } \\
& $<50$ años & $\geq 50$ años & $P$ \\
\hline E. coli & 86.7 & 83.7 & 0.32 \\
K. pneumonia & 4.3 & 5.3 & 0.56 \\
S. saprophyticus & 4 & 1.8 & 0.14 \\
Proteus mirabilis & 2.8 & 2.2 & 0.64 \\
Otros & 2.3 & 7 & 0.004 \\
\hline
\end{tabular}

Al analizar la resistencia antimicrobiana de E. coli (correspondiente a $85.5 \%$ de la muestra), encontramos diferencias significativas al comparar la resistencia antibiótica a AM $(\mathrm{p}=0.05)$ entre las 
ITU altas y bajas.

También hallamos que las mujeres de $\geq 50$ años de edad tenían mayor resistencia a ciprofloxacina $(p=0.005)$. (Tabla 3).

\begin{tabular}{|c|c|c|c|c|c|c|}
\hline \multirow{3}{*}{$\begin{array}{c}\text { Antibiótic } \\
\mathrm{o}^{*}\end{array}$} & \multicolumn{6}{|c|}{ Resistencia. n, (\%) } \\
\hline & \multicolumn{2}{|c|}{ Edad } & & \multicolumn{2}{|c|}{ Sintomatología } & \multirow{2}{*}{$\begin{array}{c}\text { Valor } \\
\text { de } \\
P\end{array}$} \\
\hline & $\begin{array}{c}<50 \text { años } \\
(\mathrm{n}=307)\end{array}$ & $\begin{array}{c}\geq 50 \\
\text { años } \\
(\mathrm{n}=189)\end{array}$ & de $P$ & $\begin{array}{c}\text { Baja } \\
(\mathrm{n}=411)\end{array}$ & $\begin{array}{c}\text { Alta } \\
(\mathrm{n}=85)\end{array}$ & \\
\hline AM & $\begin{array}{c}142 \\
(46.3)\end{array}$ & $\begin{array}{c}96 \\
(50.8)\end{array}$ & 0.33 & ${ }_{(46)} 189$ & $\begin{array}{c}49 \\
(57.7)\end{array}$ & 0.05 \\
\hline AMS & $\begin{array}{c}59 \\
(19.2)\end{array}$ & $\begin{array}{c}38 \\
(20.1)\end{array}$ & 0.81 & $75(18.3)$ & $\begin{array}{c}22 \\
(25.9)\end{array}$ & 0.11 \\
\hline CFX & $25(8.1)$ & $\begin{array}{c}22 \\
(11.6)\end{array}$ & 0.20 & $36(8.8)$ & $\begin{array}{c}11 \\
(12.9)\end{array}$ & 0.23 \\
\hline SMX & $\begin{array}{c}82 \\
(26.7)\end{array}$ & $\begin{array}{c}59 \\
(31.2)\end{array}$ & 0.28 & $110(26.8)$ & $\begin{array}{c}31 \\
(36.5)\end{array}$ & 0.07 \\
\hline NF & $2(0.7)$ & $1(0.5)$ & 0.68 & $3(0.7)$ & - & - \\
\hline CPX & $16(5.2)$ & $\begin{array}{c}23 \\
(12.2)\end{array}$ & 0.005 & $34(8.3)$ & $5(5.9)$ & 0.46 \\
\hline
\end{tabular}

Al comparar la resistencia antimicrobiana de E. coli en IU no complicadas en nuestro estudio (Hospital Privado) en relación a la obtenida en el estudio Vigí-A/SADI, observamos un aumento estadísticamente significativo en la resistencia a AM, AMS, SMX ( $p=0.04 ; p=0.01$ y $p=0.003$, respectivamente) y una disminución significativa en la resistencia a NF $(p=0.02)$. (Tabla 4; Figura1).

\begin{tabular}{|c|c|c|c|}
\hline \multirow[b]{2}{*}{ Antibiótico* } & \multicolumn{3}{|c|}{ Resistencia n, ( \%) } \\
\hline & $\begin{array}{l}\text { Vigí-A/SADI } \\
(\mathrm{n}=124)\end{array}$ & $\begin{array}{l}\% \text { de resistencia } \\
\operatorname{HP}^{* *}(\mathrm{n}=496)\end{array}$ & Valor de $P$ \\
\hline $\mathrm{AM}$ & $47(37,5)$ & $237(47,8)$ & 0,04 \\
\hline AMS & $13(10,3)$ & $97(19,6)$ & 0,01 \\
\hline $\mathrm{C} 1{ }^{\circ} \mathrm{G}$ & $17(13,9)$ & $47(9,5)$ & 0,17 \\
\hline SMX & $19(15,4)$ & $142(28,6)$ & 0,003 \\
\hline CPX & $5(3,7)$ & $40(7,9)$ & 0,12 \\
\hline NF & $3(2,5)$ & $2(0.4)$ & 0,02 \\
\hline \multicolumn{4}{|c|}{$\begin{array}{l}\text { *AM=ampicilina; } \mathrm{AMS}=\text { ampicilina-sulbactam; } \mathrm{C} 1{ }^{\circ} \mathrm{G}=\text { cefalosporinas de } \\
\text { primera generación; } \mathrm{SMX}=\text { trimetoprima-sulfametoxazol; } \\
\mathrm{CPX}=\text { ciprofloxacina; } \mathrm{NF}=\text { nitrofurantoína. }{ }^{* *} \text { Estudio actual del Hospital } \\
\text { Privado }\end{array}$} \\
\hline
\end{tabular}

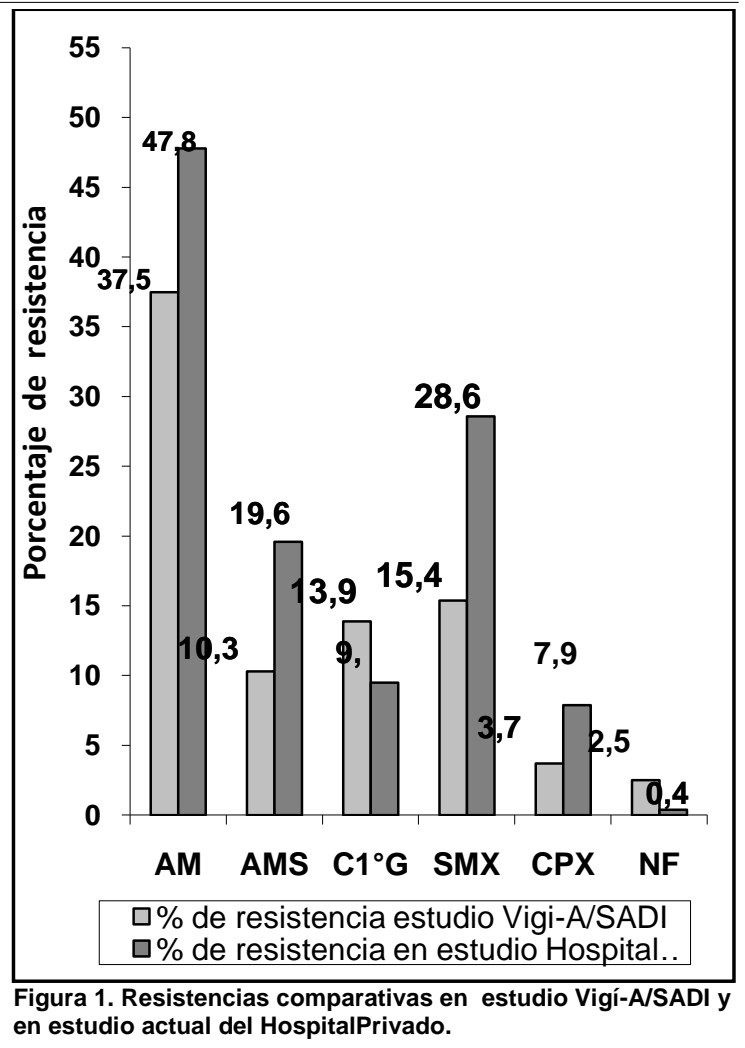

\section{Discusión}

E. coli es el microorganismo más prevalente, causando $80-85 \%$ de los episodios de IU no complicada, seguido por S. saprophyticus, K. pneumoniae y $\mathrm{P}$. mirabilis principalmente. El presente trabajo muestra que la distribución de los uropatógenos en nuestro medio es similar a los comunicados en otras publicaciones ${ }^{(4-7)}$.

Algunos expertos sugieren que las IU en mujeres postmenopáusicas sanas podrían ser incluidas dentro de las IU no complicadas dado que se infectarían por las mismas bacterias, compartirían similar perfil de resistencia, y, por ende, la evolución clínica y la respuesta al tratamiento empírico serían comparables ${ }^{(10)}$.

Por este motivo incluimos en nuestro análisis a todas las mujeres previamente sanas independientemente de su edad. Los resultados de este estudio muestran que los perfiles de fármacorresistencia de $\mathrm{E}$. coli en ambos grupos etarios son similares para todos los antibióticos excepto para CPX $(p=0.005)$. (Tabla 3$)$.

En las últimas dos décadas se observó un cambio progresivo en la resistencia antibiótica a los antimicrobianos más utilizados en la práctica clínica. Si bien los tipos de uropatógenos no han variado a lo largo del tiempo y E. coli sigue siendo la bacteria más frecuente por un amplio margen, los perfiles de resistencia han ido aumentando consistentemente, aunque existen 
diferencias notables entre las áreas geográficas analizadas $^{(4-7)}$. Estas modificaciones se observaron también en nuestra comparación con los resultados obtenidos en el estudio VigíA/SADI.

Estos cambios condujeron a una revisión de las guías de la IDSA (Infectious Disease Society of America) del año 1999 (11), con la consiguiente publicación de las modificaciones en el $2011^{(10)}$, recomendando el uso de trimetoprima o trimetoprima-sulfometoxazol como tratamiento empírico de primera línea sólo si la resistencia local era menor a $20 \%{ }^{(10)}$. Este punto de corte se basa en opinión de expertos, derivada de numerosos estudios in vitro, clínicos y de modelos matemáticos. En estas guías se resalta la importancia de conocer la prevalencia local de la resistencia de los uropatógenos, especialmente a SMX.

Los resultados de este estudio muestran que en nuestro medio la resistencia in vitro de $\mathrm{E}$. coli a SMX es elevada tanto en las cistitis como en las $\begin{array}{lllll}\text { pielonefritis } \quad(26.8 \quad \text { vs } & 36.5 & \%\end{array}$ respectivamente).Estos porcentajes de resistencia excluyen a este antibiótico como terapia empírica de primera línea a nivel local.

Las fluoroquinolonas no se recomiendan como tratamiento de elección en la mayoría de los casos de IU bajas, debido a que su uso indiscriminado trae aparejado un aumento en la resistencia y la consiguiente disminución de su eficacia tanto para las IUs como para infecciones de otra índole (p. ej. tracto respiratorio, infecciones óseas, tejidos blandos, etc.). Los resultados de este trabajo son consistentes con la literatura, donde se reporta una resistencia a ciprofloxacina en E.E.U.U. y Europa menor al $10 \%$.

Como mencionamos anteriormente, encontramos más del doble de resistencia de $\mathrm{E}$. coli a ciprofloxacina en las mujeres $\geq 50$ años comparadas con el grupo de mujeres más jóvenes (5.2 vs $12.2 \%$ respectivamente). Como en este estudio no fueron incluidas las IU recurrentes, consideramos que esta diferencia podría deberse a que las mujeres postmenopáusicas estarían expuestas en mayor proporción a fluoroquinolonas como tratamiento de otras infecciones. Esta hipótesis podría explicar también el aumento en la resistencia de uropatógenos poco frecuentes (grupo "otros") en las mujeres mayores de 50 años (2.3 vs 7; $\mathrm{p}=0.004$ ).

Con la finalidad de limitar el uso de las fluoroquinolonas y teniendo en cuenta los bajos perfiles de resistencia reportados, las guías de tratamiento vigentes y las últimas actualizaciones recomiendan el uso de nitrofurantoína como terapia de primera línea en la cistitis no complicada $(10,12)$. Numerosos estudios muestran que regímenes más convenientes que los previamente propuestos (menor duración, intervalo de dosis más espaciados) son igualmente efectivos $(13,14,15)$. La resistencia a nitrofurantoína en nuestro estudio es incluso menor a la de las fluoroquinolonas $(0.4 \%$ vs. $7.9 \%$ ), lo que la haría una opción altamente atractiva como terapia empírica de primera línea en IU bajas. El esquema de nitrofurantoína por cinco días (100 mg cada 12 horas) ha mostrado buena tolerancia y eficacia, con la ventaja de generar escasos efectos adversos ecológicos ${ }^{(1)}$. En el caso de IU altas, según nuestros resultados, recomendamos utilizar fluoroquinolonas como terapia empírica de primera línea, y evitar el uso de SMX en nuestro medio. También consideramos poner énfasis en el uso racional de antibióticos, y, en este sentido, limitar el uso indiscriminado de quinolonas en otras infecciones donde existan otras opciones terapéuticas igualmente efectivas.

Entre las características a resaltar de nuestro trabajo, podemos mencionar la importancia del conocimiento de la epidemiología local.

Entre las limitaciones, debemos destacar que sólo reportamos la prevalencia de resistencia in vitro a los antibióticos más utilizados. La tasa de fracaso terapéutico y la epidemiología de los tratamientos empíricos más utilizados en nuestro medio no fueron evaluadas. Esto constituye una aproximación microbiológica necesaria, pero no suficiente para afirmar los beneficios del uso de un determinado antibiótico en la práctica clínica. Agradecimientos por apoyo técnico: Flavia Ceballos, Paulo del Barco, Santiago Bianconi, Emanuel Saad, Domingo Balderramo.

\section{Bibliografía}

1. Hooton TM. Uncomplicated urinary tract infection. N Engl J Med. 2012;366:1028-37.

2. Foxman B. Epidemiology of urinary tract infections: incidence, morbidity, and economic costs. Am J Med 2002; 113: 5S-13S.

3. Etienne $M$, Lefebvre $E$, Frebourg $N$, Hamel $H$, Pestel-Caron M, Caron F, Bacyst Study Group. Antibiotic treatment of acute uncomplicated cystitis based on rapid urine test and local epidemiology: lessons from a primary care series. BMC Infect Dis 2014;14:137.

4. Kamenski G, Wagner G, Zehetmayer S, Fink W, Spiegel W, Hoffmann K. Antibacterial resistances in uncomplicated urinary tract infections in women: ECO.SENS II data from 
primary health care in Austria. BMC Infect Dis. 2012 Sep 18;12:222.

5. Schmiemann G, Gágyor I, Hummers-Pradier E, Bleidorn J. Resistance profiles of urinary tract infections in general practice-an observational study. BMC Urol. 2012 Nov 21;12:33.

6. Palou J, Pigrau C, Molina I, Ledesma JM, Angulo J; Etiology and sensitivity of uropathogens identified in uncomplicated lower urinary tract infections in women (ARESC Study): implications on empiric therapy. Med Clin (Barc). 2011 Jan 15;136(1):1-7.

7. Kahlmeter G. Prevalence and antimicrobial susceptibility of uncomplicated cystitis in Europe. The ECO.SENS Study. Int J Antimicrob Agents 2003; 22(Suppl. 2):49-52.

8. Rossi A, Tokumoto M, Galas $M$, Soloaga $R$, Corso $A$, et al.. Vigilancia de la resistencia a los antibacterianos en Argentina. Programa WHONET, 1995-1996. Rev Panam Salud Publica 1999;6:234-41.

9. Levy Hara G, y cols. Informe Técnico. Consenso Argentino Intersociedades para el Manejo de la Infección del Tracto Urinario - Parte I. Rev Panam Infectol 2007;9(3):57-69. Link: http://www.revistaapi.com/3\%20edicao\%202007/pdf/mat\%2010.p df.

10. Gupta K, Hooton TM, Naber KG, et al. International clinical practice guidelines for the treatment of acute uncomplicated cystitis and pyelonefritis in women: $A 2010$ update by the Infectious Diseases Society of America and the European Society for Microbiology and Infectious Diseases. Clin Infect Dis 2011; 52:e103-e120.

11. Warren JW, Abrutyn E, Hebel JR, Johnson JR, Schaeffer AJ, Satamm WE. Guidelines for antimicrobial treatment of uncomplicated acute bacterial cystitis and acute pyelonephritis in women. Infectious Diseases Society of America (IDSA). Clin Infect Dis 1999; 29:745-58.

12. The Medical Letter on Drugs and Therapeutics/February 26, 2014, Drugs for Urinary Tract Infections. JAMA 2014;311(8):855-856.

13. Knottnerus BJ, Grigoryan L, Geerlings SE, Moll van Charante EP, Verheij TJ, Kessels AG, et al. Comparative effectiveness of antibiotics for uncomplicated urinary tract infections: Network meta-analysis of randomized trials. Fam Pract. 2012;29(6):659-70.

14. Gupta K, Hooton TM, Roberts PL, Stamm WE. Short-course nitrofurantoin for the treatment of acute uncomplicates cystitis in women. Arch Intern Med 2007; 167:2207-12.

15. Christiaens TC, De Meyere M, Verschraegen G, Peersman W, Heytens S, De Maeseneer JM.
Randomised controlled trial of nitrofurantoin versus placebo in the treatment of uncomplicated urinary tract infection in adult women. Br J Gen Pract 2002;52:729-34. 